\title{
Linear Central Potential Effects Induced by Lorentz Symmetry Violation on Spin-0 Particle Under Coulomb-type scalar Potential
}

\author{
Faizuddin Ahmed ${ }^{1}$ \\ National Academy Gauripur, Assam, 783331, India
}

\begin{abstract}
In this work, quantum dynamics of a spin-0 particle under the effects of Lorentz symmetry violation in the presence of Coulombtype non-electromagnetic potential $\left(S(r) \propto \frac{1}{r}\right)$ is investigated. The non-electromagnetic (or scalar) potential is introduced by modifying the mass term via transformation $M \rightarrow M+\frac{\eta_{c}}{r}$ in the relativistic wave equation. The linear central potential induced by the Lorentz symmetry violation is a linear radial electric and constant magnetic field and, analyze the effects on the spectrum of energy and the wave function.
\end{abstract}

Keywords: Lorentz symmetry violation, Relativistic wave equations, non-electromagnetic potential, electric \& magnetic field, biconfluent Heun's function

PACS Number(s): 03.65.Pm, 11.30.Qc, 11.30.Cp

\section{Introduction}

We study motion of a relativistic spin-0 scalar particle under the Lorentz symmetry breaking effects defined by a tensor out of the Standard Model Extension (SME) $[1,2,3,4,5,6,7,8]$ in the presence of a non-electromagnetic potential inversely proportional to the axial distance $\left(\propto \frac{1}{r}\right)$. Noted that the

\footnotetext{
${ }^{1}$ faizuddinahmed15@gmail.com ; faiz4U.enter@rediffmail.com
} 
gauge sector of the Standard Model Extension consists of two classes that modifies the transport properties of space-time. These two classes are called the CPT-odd sector and the CPT-even sector (see, Refs. [1, 2, 3, 4, 5, 6, 7, 8, $9,10,11,12,13,14])$. The relativistic scalar particle under the influence of non-electromagnetic (or scalar) potential of various kinds have been studied in quantum systems $[15,16,17,18,19,20,21]$. Under the effects of Lorentz symmetry violation, the relativistic scalar particle with non-electromagnetic or electromagnetic potential have been studied in $[22,23,25,24,26,27,28$, $29,30,31,32]$.

The quantum dynamics of spin-0 scalar particle under the effects of Lorentz symmetry violation subject to a Coulomb-type non-electromagnetic potential introduced via mass transformation $M \rightarrow M+\frac{\eta_{c}}{r}$ into the wave equation is given by $[22,23,25,24,26,27,28,29,30,31,32]$

$$
p^{\mu} p_{\mu} \Psi+\frac{\alpha}{4}\left(K_{F}\right)_{\mu \nu \alpha \beta} F^{\mu \nu}(x) F^{\alpha \beta}(x) \Psi=\left(M+\frac{\eta_{c}}{r}\right)^{2} \Psi
$$

where $\alpha$ is a constant, $F_{\mu \nu}(x)$ is the electromagnetic field tensor, $\left(K_{F}\right)_{\mu \nu \alpha \beta}$ is a dimensionless tensor that governs the Lorentz symmetry violation out of the Standard Model Extension.

\section{Relativistic Scalar particle under LSV sub- ject to a non-electromagnetic Potential}

The Klein-Gordon equation under the effects of Lorentz symmetry violation defined by the Eq. (1) in the background of the Minkowski space-time is given by

$$
\begin{aligned}
& {\left[-\frac{\partial^{2}}{\partial t^{2}}+\frac{\partial^{2}}{\partial r^{2}}+\frac{1}{r} \frac{\partial}{\partial r}+\frac{\partial^{2}}{\partial z^{2}}+\frac{1}{r^{2}} \frac{\partial^{2}}{\partial \phi^{2}}\right] \Psi+\frac{\alpha}{4}\left(K_{F}\right)_{\mu \nu \alpha \beta} F^{\mu \nu}(x) F^{\alpha \beta}(x) \Psi} \\
& =\left(M+\frac{\eta_{c}}{r}\right)^{2} \Psi
\end{aligned}
$$

Using the properties of the tensor $\left(K_{F}\right)_{\mu \nu \alpha \beta}[1,2,3,4,6,7,8,11,12,26$, 
$14,22,23,25,24]$, we can rewrite (2) in the following form :

$$
\begin{aligned}
& {\left[-\frac{\partial^{2}}{\partial t^{2}}+\frac{\partial^{2}}{\partial r^{2}}+\frac{1}{r} \frac{\partial}{\partial r}+\frac{1}{r^{2}} \frac{\partial^{2}}{\partial \phi^{2}}+\frac{\partial^{2}}{\partial z^{2}}\right] \Psi} \\
& +\left[-\frac{\alpha}{2}\left(\kappa_{D E}\right)_{i j} E^{i} E^{j}+\frac{\alpha}{2}\left(\kappa_{H B}\right)_{j k} B^{i} B^{j}-\alpha\left(\kappa_{D B}\right)_{j k} E^{i} B^{j}\right] \Psi \\
& =\left(M+\frac{\eta_{c}}{r}\right)^{2} \Psi .
\end{aligned}
$$

We choose the scenario of the Lorentz symmetry violation determined by the non-null components $\left(\kappa_{D E}\right)_{11}=$ const $=\kappa_{1},\left(\kappa_{H B}\right)_{33}=$ const $=\kappa_{2}$ and $\left(\kappa_{D B}\right)_{13}=$ const $=\kappa_{3}$ with the following field configuration $[22,23,25,24$, $27,33,34]$ :

$$
\vec{B}=B_{0} \hat{z} \quad, \quad \vec{E}=\frac{\lambda}{2} r \hat{r}
$$

where $B_{0}>0, \hat{z}$ is a unit vector in the $z$-direction, $\lambda$ is a constant associated with a linear charge density of electric charges along the axial direction, and $\hat{r}$ is the unit vector in the radial direction.

Hence, equation (3) using the configuration (4) becomes

$$
\begin{aligned}
& {\left[-\frac{\partial^{2}}{\partial t^{2}}+\frac{\partial^{2}}{\partial r^{2}}+\frac{1}{r} \frac{\partial}{\partial r}+\frac{1}{r^{2}} \frac{\partial^{2}}{\partial \phi^{2}}+\frac{\partial^{2}}{\partial z^{2}}\right] \Psi} \\
& +\left[-\frac{\alpha \kappa_{1} \lambda^{2}}{8} r^{2}+\frac{\alpha \kappa_{2} B_{0}^{2}}{2}-\frac{\alpha \lambda B_{0} \kappa_{3}}{2} r\right] \Psi=\left(M+\frac{\eta_{c}}{r}\right)^{2} \Psi .
\end{aligned}
$$

Since the metric is independent of $(t, \phi, z)$, let the solution to the Eq. (6) is

$$
\Psi(t, r, \phi, z)=e^{i(-E t+l \phi+k z)} \psi(r)
$$

where $E, l$ have their usual meaning, and $k$ is a constant.

Therefore, substituting (6) into the Eq. (5), we obtain the following radial wave equation for $\psi(r)$ :

$$
\psi^{\prime \prime}(r)+\frac{1}{r} \psi^{\prime}(r)+\left[\Sigma-\frac{j^{2}}{r^{2}}-\omega^{2} r^{2}-\frac{\beta_{1}}{r}-\beta_{2} r\right] \psi(r)=0
$$


where

$$
\begin{aligned}
\Sigma & =E^{2}-M^{2}-k^{2}+\frac{1}{2} \alpha \kappa_{2} B_{0}^{2}, \\
j & =\sqrt{l^{2}+\eta_{c}^{2}}, \\
\omega & =\sqrt{\frac{1}{8} \alpha \kappa_{1} \lambda^{2}}, \\
\beta_{1} & =2 M \eta_{c}, \\
\beta_{2} & =\frac{1}{2} \alpha \lambda B_{0} \kappa_{3} .
\end{aligned}
$$

Transforming $\xi=\sqrt{\omega} r$ in the above equation (7), we have

$$
\left[\frac{d^{2}}{d \xi^{2}}+\frac{1}{\xi} \frac{d}{d \xi}+\zeta-\xi^{2}-\frac{j^{2}}{\xi^{2}}-\frac{\eta}{\xi}-\theta \xi\right] \psi(\xi)=0,
$$

where

$$
\zeta=\frac{\Sigma}{\omega} \quad, \quad \eta=\frac{\beta_{1}}{\sqrt{\omega}} \quad, \quad \theta=\frac{\beta_{2}}{\omega^{\frac{3}{2}}} .
$$

Now we impose the requirement that the wave-function $\psi(x)$ is finite both at the origin $x \rightarrow 0$ and at $x \rightarrow \infty$. Suppose the possible solution to the Eq. (9) is

$$
\psi(\xi)=\xi^{j} e^{-\frac{1}{2}(\xi+\theta) \xi} H(\xi) .
$$

Substituting the solution (11) into the Eq. (9), we obtain the following equation

$$
H^{\prime \prime}(\xi)+\left[\frac{1+2 j}{\xi}-2 \xi-\theta\right] H^{\prime}(\xi)+\left[-\frac{\tau}{\xi}+\Pi\right] H(\xi)=0,
$$

where

$$
\Pi=\zeta+\frac{\theta^{2}}{4}-2(1+j) \quad, \quad \tau=\eta+\frac{\theta}{2}(1+2 j) .
$$

Equation (12) is the biconfluent Heun's differential equation [15, 16, 17, 18, $19,20,21,24,35,36]$ with $H(\xi)$ is the Heun polynomials function.

The above equation (12) can be solved by the Frobenius method. Writing the solution as a power series expansion around the origin [37]:

$$
H(\xi)=\sum_{i=0}^{\infty} d_{i} \xi^{i}
$$


Substituting the power series solution into the Eq. (14), we obtain the following recurrence relation

$$
d_{n+2}=\frac{1}{(n+2)(n+2+2 j)}\left[\left\{\eta+\theta\left(n+\frac{3}{2}+j\right)\right\} d_{n+1}-(\Pi-2 n) d_{n}\right] .
$$

With the few coefficients are

$$
\begin{aligned}
& d_{1}=\left(\frac{\eta}{1+2 j}+\frac{\theta}{2}\right) d_{0} \\
& d_{2}=\frac{1}{4(1+j)}\left[\left\{\eta+\theta\left(j+\frac{3}{2}\right)\right\} d_{1}-\Pi d_{0}\right] .
\end{aligned}
$$

The power series expansion $H(\xi)$ becomes a polynomial of degree $n$ by imposing the following two conditions $[15,16,17,18,19,20,21,24]$

$$
\Pi=2 n, \quad(n=1,2, \ldots) \quad, \quad d_{n+1}=0 .
$$

By analyzing the first condition, we obtain following equation of the energy eigenvalue $E_{n, l}$ :

$$
\begin{aligned}
E_{n, l} & = \pm \sqrt{M^{2}+k^{2}+2 \omega\left(n+1+\sqrt{l^{2}+\eta_{c}^{2}}\right)-\frac{\beta_{2}^{2}}{4 \omega^{2}}-\frac{1}{2} \alpha B_{0}^{2} \kappa_{2}} \\
& = \pm \sqrt{M^{2}+k^{2}+|\lambda| \sqrt{\frac{\alpha}{2} \kappa_{1}}\left(n+1+\sqrt{l^{2}+\eta_{c}^{2}}\right)-\frac{1}{2} \alpha B_{0}^{2} \chi},(18
\end{aligned}
$$

where $\chi=\left[\kappa_{2}+\frac{\kappa_{3}^{2}}{\kappa_{1}}\right]$.

Note that the Eq. (18) is not the general expression of energy eigenvalue of a relativistic scalar particle. One can obtain the individual energy level and the eigenfunction one by one by imposing the additional recurrence condition $d_{n+1}=0$ on the eigenvalue problem.

The corresponding wave-functions are given by

$$
\psi_{n, l}(\xi)=\xi^{\sqrt{l^{2}+\eta_{c}^{2}}} e^{-\frac{1}{2}\left[\xi+\frac{\beta_{2}}{\omega^{\frac{3}{2}}}\right] \xi} H(\xi) .
$$


Now, we evaluate the individual energy levels and the eigenfunctions one by one as done in [21]. For example, $n=1$, we have $\Theta=2$ and $d_{2}=0$ which implies

$$
\begin{aligned}
& \frac{2}{\eta+\theta\left(\frac{3}{2}+j\right)} d_{0}=\left(\frac{\eta}{1+2 j}+\frac{\theta}{2}\right) d_{0} \\
& \Rightarrow \lambda_{1, l}=\left[\frac{2 M^{2} \eta_{c}^{2}}{\sqrt{\frac{\alpha}{2} \kappa_{1}}\left(\frac{1}{2}+j\right)}+\frac{4 M \eta_{c} B_{0} \kappa_{3}}{\kappa_{1}}\left(\frac{1+j}{\frac{1}{2}+j}\right)+\frac{4 B_{0}^{2} \kappa_{3}^{2} \sqrt{\frac{\alpha}{2}}}{\kappa_{1}^{\frac{3}{2}}}\left(\frac{3}{2}+j\right)\right] .
\end{aligned}
$$

a constraint on the linear charge density $\lambda_{1, l}$. We can see from Eq. (20), that the allowed values of the linear charge density depends on the quantum numbers of the system $\{n, l\}$, the parameters $\left\{\alpha, B_{0}, \kappa_{1}, \kappa_{2}, \kappa_{3}\right\}$ associated with the background of Lorentz Symmetry Violation, and Coulomb-type potential.

Therefore, the ground state energy level for the radial mode $n=1$ is given by

$$
E_{1, l}= \pm \sqrt{M^{2}+k^{2}+\lambda_{1, l} \sqrt{\frac{\alpha}{2} \kappa_{1}}\left(2+\sqrt{l^{2}+\eta_{c}^{2}}\right)-\frac{1}{2} \alpha B_{0}^{2} \chi}
$$

And the ground state eigenfunction is

$$
\psi_{1, l}(\xi)=\xi^{\sqrt{l^{2}+\eta_{c}^{2}}} e^{-\frac{1}{2}\left[\xi+\sqrt{\frac{2 \alpha}{\omega_{1, l} \kappa_{1}}} B_{0} \kappa_{3}\right] \xi}\left(1+d_{1} \xi\right),
$$

where we have chosen $d_{0}=1$ and

$$
d_{1}=\frac{1}{\sqrt{\omega_{1, l}}}\left[\frac{M \eta_{c}}{\left(\frac{1}{2}+\sqrt{l^{2}+\eta_{c}^{2}}\right)}+\sqrt{\frac{\alpha}{2 \kappa_{1}}} B_{0} \kappa_{3}\right] \quad, \quad \omega_{1, l}=\lambda_{1, l} \sqrt{\frac{\alpha}{8} \kappa_{1}} .
$$

We can see that the lowest energy state (21) plus the ground state wave function (22)-(23) with the restriction on the linear charge density (20) is defined for the radial mode $n=1$, instead of $n=0$. This effect arises due to the presence of Coulomb-type potential and the Lorentz symmetry violation. 


\section{Conclusions}

We have investigated the effects of a linear central potential induced by the violation of Lorentz symmetry breaking background on a relativistic scalar particle subject to Coulomb-type non-electromagnetic potential. we have shown that the bound states solution to the modified Klein-Gordon equation can be obtained in a possible scenario of the Lorentz symmetry breaking effects defined by a linear radial electric field produced by linear distribution of electric charges, a uniform magnetic along the $z$-direction, a tensor background that governs the Lorentz symmetry violation possessing nonnull components $\left(\kappa_{D B}\right)_{13}=$ const, $\left(\kappa_{D E}\right)_{11}=$ const and $\left(\kappa_{H B}\right)_{33}=$ const subject to a Coulomb-type non-electromagnetic (or scalar) potential. The radial equation of the relativistic wave equation is derived and for a suitable wave function, the binconfluent Heun differential equation is achieved. Using the power series solution, this Heun equation is solved and truncated this power series by imposing two conditions simultaneously. After analysing the truncating conditions, the non-compact expression of the energy eigenvalues (18) and the wave function (19) is obtained. By imposing the additional recurrence condition $d_{n+1}=0$ on the eigenvalue problem, one can obtain the individual energy levels and the wave function, for example, the lowest state energy level (21) and the corresponding wave function (22)-(23) with the restriction (20) on the linear charge density parameter for the radial mode $n=1$ instead of $n=0$. This effect arises due to the presence of Coulombtype scalar potential and Lorentz symmetry violation background. We have seen a quantum effect due to the dependence of the linear charge density on the quantum numbers $\{n, l\}$ of the system, so we have labelled $\lambda \rightarrow \lambda_{n, l}$. In addition, this linear charge density depends on the Lorentz symmetry breaking parameters $\left\{\alpha, B_{0}, \kappa_{1}, \kappa_{2}, \kappa_{3}\right\}$ as well as on the Coulomb-potential. 


\section{Conflict of Interest}

There is no conflict of interest regarding publication this paper.

\section{Data Availability}

No data has been used to prepare this paper.

\section{References}

[1] D. Colladay and V. A. Kostelecky, Phys. Rev. D 55, 6760 (1997).

[2] D. Colladay and V. A. Kostelecky, Phys. Rev. D 58, 116002 (1998).

[3] S. R. Coleman and S. L. Glashow, Phys. Rev. D 59, 116008 (1999).

[4] V. A. Kostelecky and S. Samuel, Phys. Rev. Lett. 63, 224 (1989).

[5] V. A. Kostelecky and S. Samuel, Phys. Rev. Lett. 66, 1811 (1991).

[6] V. A. Kostelecky and S. Samuel, Phys. Rev. D 39, 683 (1989).

[7] V. A. Kostelecky and S. Samuel, Phys. Rev. D 40, 1886 (1989).

[8] V. A. Kostelecky and R. Potting, Nucl. Phys. B 359, 545 (1991); Phys. Lett. B 381, 89 (1996); Phys. Rev. D 51, 3923 (1995).

[9] A.P. Baeta Scarpelli, H. Belich, J. L. Boldo, L. P. Colatto, J. A. HelayelNeto, and A. L. M. A. Nogueira, Nucl. Phys. B (Proc. Suppl.) 127, 105 (2004).

[10] S. Carroll, G. Field and R. Jackiw, Phys. Rev. D 41, 1231 (1990).

[11] V. A. Kostelecky and M. Mewes, Phys. Rev. Lett. 87, 251304 (2001).

[12] V. A. Kostelecky and M. Mewes, Phys. Rev. D 66, 056005 (2002). 
[13] V. A. Kostelecky and M. Mewes, Phys. Rev. Lett. 97, 140401 (2006).

[14] H. Belich, F. J. L. Leal, H. L. C. Louzada and M. T. D. Orlando, Phys. Rev. D 86, 125037 (2012).

[15] M. Hosseini, H. Hassanabadi, S. Hassanabadi and P. Sedaghatnia, Int. J. Geom. Meths. Mod. Phys. 16, 1950054 (2019).

[16] M. de Montigny, M. Hosseinpour and H. Hassanabadi, Int. J. Mod. Phys. A 31, 1650191 (2016).

[17] M. S. Cunha, C. R. Muniz, H. R. Christiansen and V. B. Bezerra, Eur. Phys. J. C 76, 512 (2016).

[18] F. Ahmed, Adv. High Energy Phys. 2020, 4832010 (2020).

[19] H. Hassanabadi, E. Maghsoodi and S. Zarrinkamar, Ann. Phys. (Berlin) 525, 944 (2013).

[20] M. Hosseinpour, H. Hassanabadi and Marc de Montigny, Int. J. Geom. Meths Mod. Phys. 15, 1850165 (2018).

[21] E. R. F. Medeiros and E. R. Bezerra de Mello, Eur. Phys. J. C 72, 2051 (2012).

[22] K. Bakke and H. Belich, Ann. Phys. (N. Y.) 354, 1 (2015).

[23] K. Bakke and H. Belich, Ann. Phys. (N. Y.) 373, 115 (2016).

[24] F. Ahmed, IJMPA (2021), DOI:10.1142/S0217751X21501281.

[25] R. L. L. Vitoria and H. Belich, Eur. Phys. J. C 78, 999 (2018).

[26] R. L. L. Vitoria, H. Belich and K. Bakke, Adv. High Energy Phys. 2017, 6893084 (2017).

[27] F. Ahmed, DOI : 10.20944/preprints202105.0782.v1. 
[28] K. Bakke and H. Belich, Ann. Phys. (N. Y.) 360, 596 (2015).

[29] R. L. L. Vitoria, K. Bakke and H. Belich, Ann. Phys. (N. Y.) 399, 117 (2018).

[30] F. Ahmed, DOI : 10.20944/preprints202105.0755.v1.

[31] R. L. L. Vitoria, H. Belich and K. Bakke, Eur. Phys. J. Plus 132, 25 (2017).

[32] F. Ahmed, DOI : 10.20944/preprints202105.0748.v1.

[33] M. Ericsson and E. Sjoqvist, Phys. Rev. A 65, 013607 (2001).

[34] I. C. Fonseca and K. Bakke, J. Math. Phys. 56, 062107 (2015).

[35] A. Ronveaux, Heun's Differential Equations, Oxford University Press, Oxford ( 1995).

[36] S. Y. Slavyanov and W. Lay, Special Functions: A Unified Theory Based in Singularities, Oxford University Press, New York (2000).

[37] G. B. Arfken and H. J. Weber, Mathematical Methods For Physicists, Elsevier Academic Press, London (2005). 